\title{
EVALUACIÓN DE DAÑO CAUSADO POR Prodiplosis longifila \\ GAGNÉ (DIPTERA: Cecidomyiidae) EN UN CULTIVO DE Solanum \\ lycopersicum L. EN GIGANTE, HUILA
}

\author{
Oscar Valbuena Calderón ${ }^{1}$, Adriana Bolaños Trujillo², Marion Muriel Rodriguez ${ }^{3}$ \\ RESUMEN
}

El estudio tuvo como objetivo evaluar la severidad del daño causado por Prodiplosis. longifila Gagné (Diptera: Cecidomyiidae) en diferentes tratamientos de control en un cultivo de tomate en el municipio de Gigante-Huila, en donde debido al incremento de área sembrada en cultivos de tomate (Solanum lycopersicum L.), el daño causado por $P$. longifila ha sido limitante para el desarrollo del cultivo en la zona. Se establecieron tres tratamientos de control los cuales fueron: T1) aplicación de insecticida de origen vegetal; T2) aplicación de 4 insecticidas de síntesis química más uno de origen vegetal; T3) aplicación de 4 insecticidas de síntesis química; y un Testigo donde no se realizaron aplicaciones. Posteriormente fueron evaluadas tres variables: 1) número de larvas vivas; 2) presencia/ausencia del daño y del insecto; y 3) número total de frutos sanos y afectados; y los resultados fueron analizados mediante ANAVA y separación de medias LSD Fisher con Infostat 2017. El crecimiento más elevado de larvas vivas fue para el Testigo y el más bajo fue para T2 y T3; en la variable presencia/ausencia no existió diferencias significativas entre los tratamientos y el testigo; sin embargo, en la variable de frutos sanos/afectados se encontraron 2 grupos con diferencias significativas, evidenciando que T2 y T3, presentaron un mayor control del insecto. Este estudio permitió evidenciar que el Testigo y T1 fueron diferentes a los T2 y T3, siendo estos últimos más efectivos en la disminución del daño causado por P. longifila.

Palabras claves: Caracha, Control Químico, Control Natural, Plaga, Tomate.

DOI: https://doi.org/10.22490/26653176.2344

\footnotetext{
${ }^{1}$ Magister, Docente, UNAD, CCAV Pitalito, Programa Agronomía, oscar.valbuena@unad.edu.co

2 Estudiante, UNAD, CCAV Pitalito, Programa Agronomía, adriana9812@gmail.com

${ }^{3}$ Estudiante, UNAD, CCAV Pitalito, Programa Agronomía, mayiroth@yahoo.es
} 


\begin{abstract}
The municipality of Gigante, Huila, Colombia has an estimated agricultural production that accounts for over 38.000 tons/year, where Solanum lycopersicum L, is highlighted; however due to the annual cultivated area increase, phytosanitary issues have been a restriction for higher yields. One of the major problems is the attack of caracha Prodiplosis longifila Gagné (Diptera: Cecidomyiidae). The aim of this study was to evaluate the severity of the damage caused by $P$. longifila in different control treatments in one tomato crop in the municipality of Gigante, Huila. Three different control treatments where established: T1) application of a vegetal insecticide; T2), application of 4 synthetic chemical insecticide and one vegetal insecticide; T3) application of 4 synthetic chemical insecticides, and a Control where no applications where conducted against the insect. After that, three variables were evaluated: 1) number of alive larvae; 2) presence/absence of the insect damage; and 3) total number of attacked and non-attacked fruits. The highest growth of alive larvae was for the Control and the lowest was for T2 and T3; in presence/absence variable there was no significant difference between treatments and Control; however, in variable attacked/not-attacked fruits 2 groups with significant differences were found, showing that T2 and T3, presented a greater control of the insect. This study showed that Control and T1 where different than T2 and T3, where these last two were more effective in decreasing the damage of caused by P. longifila.
\end{abstract}

Key-words: Chemical Control, Natural Control, Insect Population Growth. 


\section{INTRODUCCIÓN}

El municipio de Gigante, Huila, Colombia tiene una producción agropecuaria de más de 38.000 ton/año, donde se destaca el cultivo de tomate Solanum lycopersicum L. (Sanmiguel, 2016); sin embargo debido al incremento anual del área sembrada de este cultivo los problemas fitosanitarios han sido limitantes hasta en un 80\% en la obtención de rendimientos (Bolaños, 2016; Rivera, 2016).

Actualmente se evidencia una disminución de los rendimientos en la producción por el daño causado por P. longifila, así como un aumento en los costos de producción, por lo cual los agricultores de la zona tienen una menor estabilidad económica, generando mayor pobreza y desplazamiento de este cultivo hacia otras zonas del Departamento (Rincón, 2016). En el año 2013, se presentó por primera vez la afectación, considerándose actualmente como la mayor limitante en producción (Bolaños, 2016; Rivera, 2016).

La contaminación y la alteración del ecosistema son factores preocupantes por el incremento en las aplicaciones de insecticidas para lograr controlar y disminuir las afectaciones de esta especie, pasando de un solo ingrediente activo (Spirotetramat) y una sola aplicación durante el ciclo de cultivo, a realizar aplicaciones de hasta 8 productos con diferentes dosis: Spinetoram en dosis de 10cc, Cipermetrina en dosis de 20cc, Fipronil en dosis de 24cc, Abamectina en dosis de 25cc, (Fernández-Kohatsu., 2016) (Cardona, et al, 2010) Tiametoxan-Lambdacihalotrina dosis de 20cc (Cardona, et al, 2010) Spirotetramat en dosis de 200cc, Flubendiamida en dosis de 10cc, Deltametrin en dosis de 20cc, Imidacloprid en dosis de 20cc (Fernández-Kohatsu., 2016; Cardona, et al, 2010), cada 4 días (Lozano, 2016).

La salud de los trabajadores también se ve afectada negativamente, debido a que el uso excesivo de insecticidas constituye uno de los principales riesgos profesionales, puesto que estos provocan 
intoxicación y muerte, y en algunos casos, cáncer profesional y trastornos de la función reproductora (OIT, 2000).

Los tratamientos de síntesis química de uso convencional en el control de $P$. longifila han disminuido su efecto contra la plaga, y se encuentran productos de origen natural para su control, los cuales pueden convertirse en una alternativa para el control del insecto; por tanto, toma importancia evaluar la severidad del daño causado por $P$. longifila en diferentes tratamientos de control en Solanum lycopersicum, así como estimar el daño causado por la $P$. longifila y establecer el tratamiento más efectivo para su control.

\section{MATERIALES Y MÉTODOS}

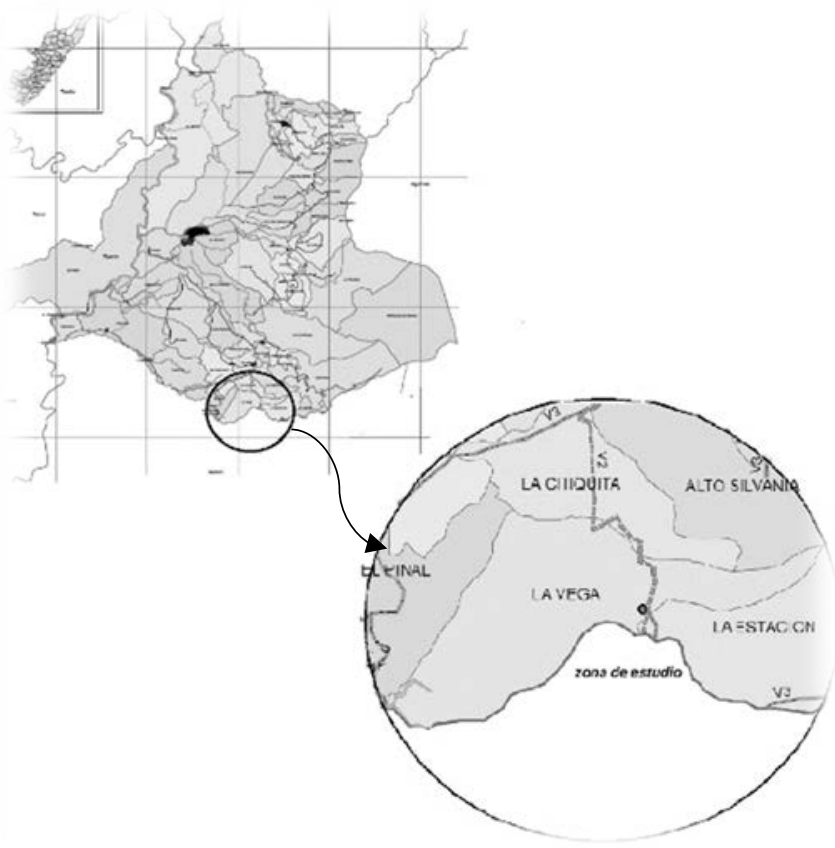

Figura 1. Localización sitio de estudio.

El estudio se realizó en la vereda la Vega del municipio de Gigante en el departamento del Huila (Latitud 2¹6'4.16" N; Longitud 75³0'16.04" O), ubicada a $1470 \mathrm{msnm}$, con una temperatura promedio de $20^{\circ} \mathrm{C}$, una precipitación media anual de $2200 \mathrm{~mm}$ y humedad relativa 
del 80\% (Fig. 3). Según el IGAC, se caracteriza por tener suelos superficiales a moderadamente profundos, bien drenados, ácidos a ligeramente ácidos, de fertilidad baja a moderada, se ubica en la Categoría IV: tierras ligeramente inclinadas a moderadamente quebradas, localizadas en clima medio y húmedo en todos los paisajes. En esta zona las tierras se usan principalmente para explotaciones agrícolas (café, frutales, entre otros). Para la evaluación de las variables se realizó un diseño de bloques completos al azar (BCA) con 3 tratamientos x 4 repeticiones +1 testigo, con igual número de repeticiones, lo cual arroja el siguiente modelo estadístico:

$$
\mathbf{Y}_{\mathbf{i j}}=\boldsymbol{\mu}+\mathbf{T} \mathbf{p}_{\mathbf{i}}+\mathbf{E r}_{\mathbf{i j}}
$$

Donde, $\mathrm{Y}_{\mathrm{ij}}=$ variable de daño causado por $P$. longifila en el i-ésimo tratamiento + testigo $\mu=$ media general $\mathrm{Tp}_{\mathrm{i}}=$ efecto de los tratamientos Er= error experimental

Los tratamientos y el testigo que se evaluaron fueron: Testigo) No se realizaron aplicaciones de ningún tipo contra el insecto; Tratamiento 1) Aplicación con un insecticida de origen vegetal, cada 7 días, durante 4 meses; Tratamiento 2) Aplicación de 4 insecticidas de síntesis química y un insecticida de origen vegetal cada 7 días, durante un periodo de 4 meses; Tratamiento 3) Aplicación de 4 insecticidas de síntesis química cada 7 días (Tabla 2). Las aplicaciones de los tratamientos se realizaron en los diferentes estados fenológicos de las plantas de tomate, en la etapa vegetativa se efectuaron 5 aplicaciones, en la floración se contaron con 6 aplicaciones y en la etapa de fructificación se realizaron 8 aplicaciones respectivamente. 
Tabla 1. Descripción de productos de síntesis química y de origen vegetal que fueron usados para controlar el daño causado por P. longifila en el cultivo de tomate, en el municipio de Gigante, Huila.

\begin{tabular}{|c|c|c|c|c|c|}
\hline Trat. & Insecticida & $\begin{array}{l}\text { Dosis } \\
\text { X 20L }\end{array}$ & Ingrediente activo & Concentración & $\begin{array}{l}\text { Categoría } \\
\text { toxicológica }\end{array}$ \\
\hline $\mathrm{T} 1$ & Capsialil $®$ & $40 \mathrm{cc}$ & $\begin{array}{l}\text { Extractos de ajo } \\
\text { Extracto de ají }\end{array}$ & $\begin{array}{l}542 \mathrm{~g} / \mathrm{L} \\
434 \mathrm{~g} / \mathrm{L}\end{array}$ & $\begin{array}{l}\text { III medianamente } \\
\text { tóxico }\end{array}$ \\
\hline \multirow{5}{*}{$\mathrm{T} 2$} & Trigard® & $6 \mathrm{gr}$ & Ciromazina & $750 \mathrm{~g} / \mathrm{kg}$ & \multirow{5}{*}{$\begin{array}{l}\text { IV ligeramente tóxico } \\
\text { III medianamente } \\
\text { tóxico } \\
\text { II moderadamente } \\
\text { peligroso } \\
\text { III. Ligeramente } \\
\text { Peligroso. } \\
\text { III medianamente } \\
\text { tóxico }\end{array}$} \\
\hline & Referi ${ }^{\circledR}$ & 20сc & Fipronil & $200 \mathrm{~g} / \mathrm{L}$ & \\
\hline & Engeo ${ }^{\circledR}$ & 20сc & $\begin{array}{c}\text { Tiametoxam } \\
\text { Lambda-cihalotrina }\end{array}$ & $\begin{array}{l}141 \mathrm{~g} / \mathrm{L} \\
106 \mathrm{~g} / \mathrm{L}\end{array}$ & \\
\hline & Coragen ${ }^{\circledR}$ & $10 \mathrm{cc}$ & Chlorantraniliprole & $184 \mathrm{~g} / \mathrm{L}$ & \\
\hline & Capsialil ${ }^{\circledR}$ & $40 \mathrm{cc}$ & $\begin{array}{l}\text { Extractos de ajo } \\
\text { Extracto de ají }\end{array}$ & $\begin{array}{l}542 \mathrm{~g} / \mathrm{L} \\
434 \mathrm{~g} / \mathrm{L}\end{array}$ & \\
\hline \multirow{4}{*}{ T3 } & Trigard $\AA$ & $6 g r$ & Ciromazina & $750 \mathrm{~g} / \mathrm{kg}$ & $\begin{array}{l}\text { IV ligeramente tóxico } \\
\text { III medianamente }\end{array}$ \\
\hline & Referi ${ }^{\circledR}$ & $20 \mathrm{cc}$ & Fipronil & $200 \mathrm{~g} / \mathrm{L}$ & tóxico \\
\hline & Engeo ${ }^{\circledR}$ & $20 \mathrm{cc}$ & $\begin{array}{c}\text { Tiametoxam } \\
\text { Lambda-cihalotrina }\end{array}$ & $\begin{array}{l}141 \mathrm{~g} / \mathrm{L} \\
106 \mathrm{~g} / \mathrm{L}\end{array}$ & $\begin{array}{l}\text { II moderadamente } \\
\text { peligroso }\end{array}$ \\
\hline & Coragen $\AA$ & $10 \mathrm{cc}$ & Chlorantraniliprole & $184 \mathrm{~g} / \mathrm{L}$ & $\begin{array}{l}\text { III. Ligeramente } \\
\text { Peligroso. }\end{array}$ \\
\hline
\end{tabular}

Durante el ciclo de cultivo se realizaron evaluaciones a cada una de los tratamientos en el cual se evaluó: 1) número de larvas vivas: se sumó el número de larvas vivas por cada parcela y se calculó el promedio con las 4 repeticiones.; 2) presencia/ausencia del daño o insecto (Posada, 2016): los indicadores fueron la necrosis en el borde de las hojas (Fig. 2C) y/o la presencia del estado larval del insecto. Los datos recolectados en las tablas de campo fueron valorados de acuerdo a la ausencia o presencia; por tanto, se tomó como 1 el valor de presencia y 0 el valor de ausencia, de manera que pudiera generarse un indicador numérico que permitiera el análisis cuantitativo de la información; 3) número total de frutos sanos y afectados por P. longifila: el indicador fue la presencia de costras o cicatrices en los frutos (Fig. 2A). Las variables 1 y 3 fueron 
evaluadas de acuerdo al manual para la elaboración de protocolos para ensayos de eficacia de la ANDI (2015), mientras que la variable 2 fue tomada del método de evaluación de (Chaves Vergara, 2002) en su estudio de la dinámica poblacional de la P. longifila, el cual también utilizó la variable de larvas vivas.

La primera evaluación se realizó un día antes de la primera aplicación, luego se hizo una evaluación a las 72 horas después de cada aplicación y la última evaluación se realizó 3 días después de la última aplicación. El análisis se realizó con el programa estadístico Infostat versión 2017, con el cual se realizó un análisis univariado ANOVA, SC tipo III con el método de comparación LSD (Least Significant Difference) Fisher, en lista descendente y a un nivel de significancia de $\mathrm{p}<0.05$, en donde para cada uno de los datos analizados se estructuró un nivel de confianza estándar del 95\% (Di Rienzo et al, 2017). Se realizaron 20 evaluaciones, es decir durante 20 semanas hasta el 30 de mayo, para determinar el número de larvas vivas en las parcelas de los tratamientos 1, 2 y 3; y en el testigo se realizaron 17 evaluaciones hasta el 10 de mayo, debido a que las plantas no presentaban frutos y estaban totalmente afectadas por la plaga.

La semilla que se utilizó fue híbrido conquistador (Semprecol) adquirida en el almacén AGROSOYA, Pitalito, en invernadero tipo capilla de dos naves, en madera con plástico calibre 6, en un área de $320 \mathrm{~m} 2$, las parcelas fueron de $20 \mathrm{~m} 2$ con una densidad de siembra de 2,6 plantas/m2. Distancias de siembra: a doble surco o doble hilera a una distancia de 0,4 x 0,5 mts entre plantas y 1,5 mts entre calles. El sistema de riego utilizado fue por goteo con cintas.

Para el control de enfermedades, en el suelo se realizaron aplicaciones preventivas para Fusarium sp., Erwinia sp. y Nemátodos. En las hojas se realizaron controles preventivos para gota (Phytophthora infestans) y aplicaciones a la aparición de los síntomas para demás enfermedades. La fertilización se realizó por el riego, y se empezó a los 8 días del trasplante. 
El equipo que se utilizó fue Bomba de espalda marca Royal Cóndor de 20lt, presión de 40 psi, boquilla cono hueco Albuz lila HC 0075, el agua fue tomada del distrito de riego (Asopiñal), con pH 5,5 y con un volumen final de la mezcla es de 400 l/has, las aplicaciones se realizaron de abajo hacia arriba, detenidamente con la intención de garantizar el cubrimiento y la llegada del producto al insecto. Se dirigió principalmente a los cogollos ya que es el lugar de mayor presencia de las larvas de $P$. longifila, el mismo día, con el mismo equipo y la misma persona para eliminar error estadístico.

\section{RESULTADOS Y DISCUSIÓN}

Como se muestra en la figura 2, el crecimiento más elevado en la curva de comportamiento fue para el testigo y el más bajo fue para los tratamientos con productos de síntesis química; en la figura 4, se observa como el crecimiento del promedio de larvas vivas es similar entre el testigo y el tratamiento 1 hasta la semana número 11, de ahí en adelante el testigo creció de forma más elevada, aunque con un comportamiento similar.

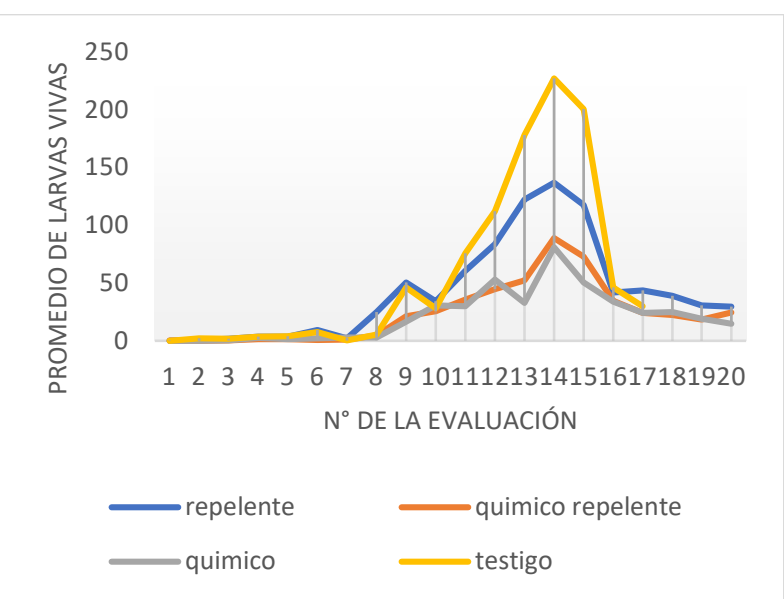

Figura 2. Comportamiento de la población de $P$. longifila en los tratamientos y testigo en cada una de las etapas fenológicas.

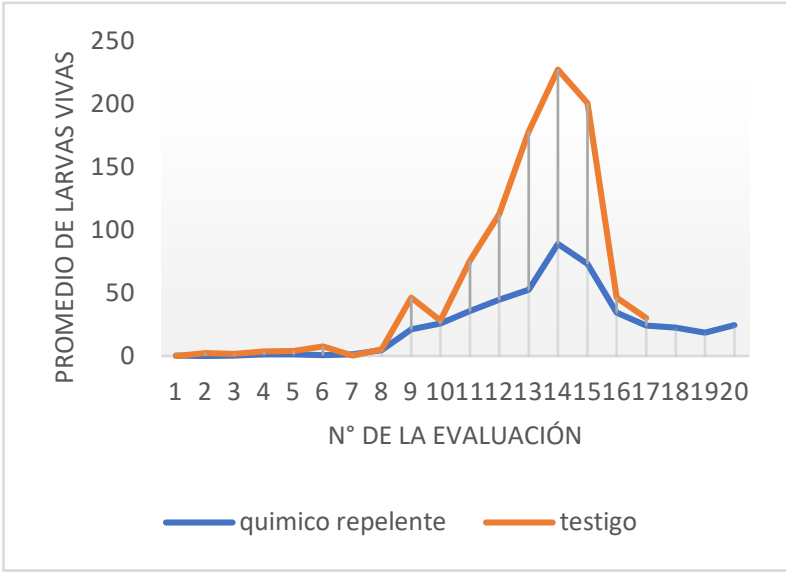

Figura 3. Comportamiento de la población de P. longifila en el tratamiento 2 vs. testigo. 


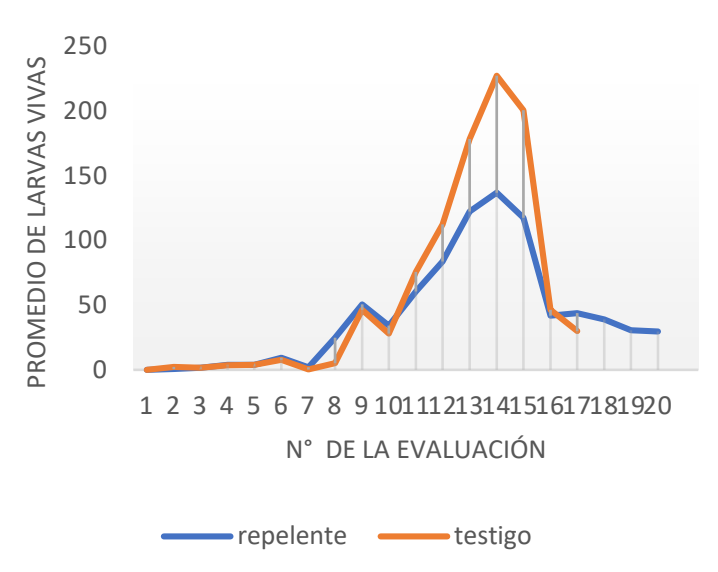

Figura 4. Comportamiento de la población de P. longifila en el tratamiento 1 vs. testigo.

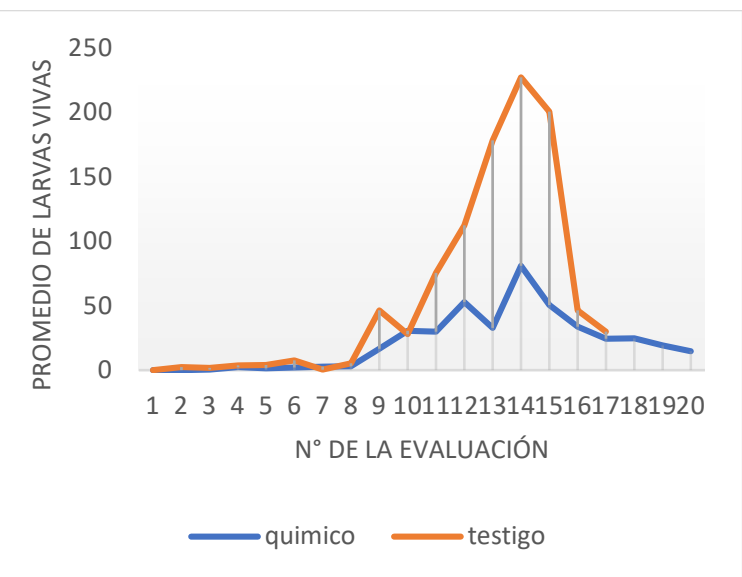

Figura 5. Comportamiento de la población de P. longifila en el tratamiento 3 vs. testigo.

En las figuras 3 y 5, se observa como los tratamientos en los que se utiliza insecticidas de origen químico a partir de la semana 10 tienen un crecimiento discreto en comparación del testigo. Las poblaciones de larvas vivas de $P$. longifila fueron menores en los tratamientos químicos al igual que en Lodana y Estancia Vieja (Ecuador) como lo reporta Valarezo et al. (2003), donde determinaron que las poblaciones fueron menores debido al control químico realizado por los agricultores de la zona.

De acuerdo a lo reportado por Diaz-Silva (2011), el comportamiento en crecimiento de la población fue muy complejo y debido a las características estáticas de esta evaluación, los valores se representan de manera lineal sobre cada uno de los tratamientos. Sin embargo, los resultados arrojaron comportamientos similares a los esperados frente al Testigo, teniendo en cuenta que la no aplicación de productos genera una mayor susceptibilidad para que el tomate sea identificado como hospedero de este insecto plaga.

Como se muestra en la Tabla 2, el análisis estadístico arrojó un coeficiente de variación del 59,06\%, con 3 grados de libertad para el modelo, un F calculado de 1,01 y valor P de 0,3898, es 
decir mayor a 0,05, por tanto, no se encuentra el nivel de confianza necesario para garantizar las diferencias significativas entre las medias analizadas.

Como se puede evidenciar en otros estudios como Cardona et al. (2016), se estableció un lote de $1000 \mathrm{~m}^{2}$ con bloques de $500 \mathrm{~m}^{2}$ por cada tratamiento, y de igual manera se realizaron las observaciones sobre el nivel de daño del insecto sobre el cultivo; siendo similar al diseño experimental planteado para este estudio.

Tabla 2. Análisis de varianza tabla (Parcial SS) para la variable de ausencia/presencia.

\begin{tabular}{|c|c|c|c|c|c|}
\hline Variable & $\mathbf{N}$ & & $\mathbf{R}^{2}$ & Adj $\mathbf{R}^{2}$ & $\mathrm{CV}$ \\
\hline Ausencia & encia & & 0,01 & $7,4 \mathrm{E}-05$ & 59,06 \\
\hline S.V. & SS & df & MS & $\mathbf{F}$ & p-value \\
\hline Model. & 4945,73 & 3 & 1648,58 & 1,01 & 0,3898 \\
\hline TRATA & 4945,73 & 3 & 1648,58 & 1,01 & 0,3898 \\
\hline Error & 484429,27 & 296 & 1636,59 & & \\
\hline Total & 489375,00 & 299 & & & \\
\hline
\end{tabular}

Aunque se observan diferencias en el comportamiento de la variable para cada uno de los tratamientos, y en comparación con el Testigo, la separación de medias por LSD Fisher $(\mathrm{p}<0,05)$ indica que no existen diferencias significativas, entre los tratamientos y el control (Figura 6), lo cual indica que independientemente del tratamiento que se aplique o inclusive sin la aplicación de alguno de ellos, el avance en el daño evidenciado mediante la variable Ausencia/Presencia de $P$. longifila, continúa su escala de evolución por encima del tipo de control que puedan ejercer los productos utilizados en este estudio. 


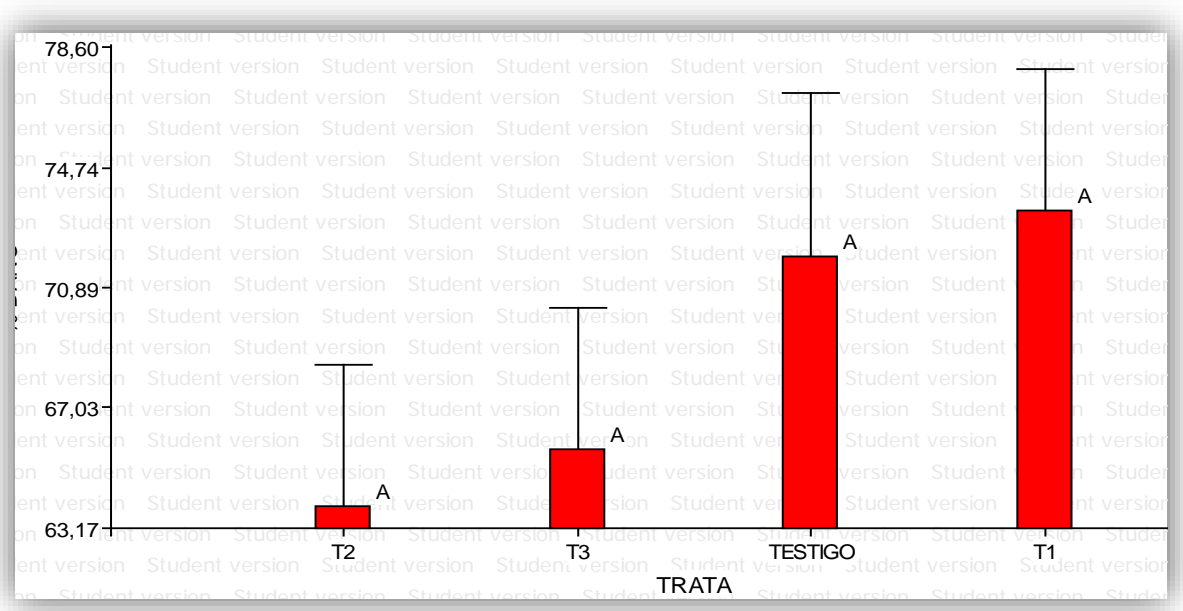

Figura 6. Comportamiento estadístico - variable ausencia/presencia de P. longifila en los diferentes tratamientos aplicados en el cultivo de tomate, en el municipio de Gigante, Huila.

Estos resultados coinciden con los reportados por Cardona et al. (2016), en donde no se encontró diferencia significativa entre los tratamientos con productos de síntesis química y otros de origen natural, medidos en nivel de infestación dentro de los cultivos en un arreglo espacial similar al planteado en este estudio.

Tal como se muestra en la tabla 3, el Tratamiento 1 (repelente) y el testigo no presentan diferencias significativas entre sí, por tanto, fueron separados con valor de la misma letra "A”; de igual forma los tratamientos 2 (químico + repelente) y tratamiento 3 (químico) no presentaron diferencias significativas entre sí, por tanto, fueron separados en otro conjunto con valor de letra "B". El análisis estadístico arrojó un coeficiente de variación del 85,053\%, con 3 grados de libertad para el modelo, un F calculado de 12,95 y valor P de 0,0001. 
Tabla 3. Análisis de la Varianza (SC tipo III) variable de frutos sanos y afectados

\begin{tabular}{lccccc} 
Variable & $\mathbf{N}$ & \multicolumn{2}{c}{$\mathbf{R}^{2}$} & $\mathbf{R}^{\mathbf{2}} \mathbf{A j}$ & $\mathbf{C V}$ \\
\hline FS & 150 & & 0,21 & 0,19 & 85,53 \\
\hline F.V. & SC & gl & $\mathbf{C M}$ & $\mathbf{F}$ & p-valor \\
\hline Modelo. & 55166,83 & 3 & 18388,94 & 12,95 & $<0,0001$ \\
TRATA & 55166,83 & 3 & 18388,94 & 12,95 & $<0,0001$ \\
Error & 207266,74 & 146 & 1419,64 & & \\
Total & 262433,57 & 149 & & & \\
TRATAM & Medias & $\mathbf{n}$ & E.E. & \\
T1 & 18,45 & 40 & 5,96 & A \\
TESTIGO & 32,37 & 30 & 6,88 & A \\
T2 & 57,33 & 40 & 5,96 & B \\
T3 & 65,15 & 40 & 5,96 & B
\end{tabular}

El mayor número de frutos afectados por esta plaga se observa en el testigo al igual que lo reportado por Rendón (2015), siendo estadísticamente diferente a los tratamientos químicos, así como la utilización de estos permitió obtener un mayor número de frutos sanos. El daño que causa P. longifila es tan severo que al no tratarse y al no bajar al mínimo los niveles de su población causa detenimiento del crecimiento de la planta tal como lo expresa Sarmiento (1997) ya que dañan los tejidos epidérmicos de los brotes nuevos de la planta y flores, produciendo el daño parcial y/o total de las mismas. En este caso también fue evidente el daño evaluado en los frutos, al encontrar que inclusive en un menor número de semanas de toma de datos, dado el avanzado estado de deterioro de la planta para el Tratamiento 1 y el Testigo, se encontró una mayor incidencia de daño que en los Tratamientos 2 y 3, durante las etapas tempranas de fructificación, pues como lo menciona Diaz-Silva (2011), las larvas se alojan en los sépalos y pueden esperar la formación de fruto para su consumo. 


\section{CONCLUSIONES}

No existe diferencia significativa en la severidad del daño causado por P. longifila cuando se analizan las variables de crecimiento de la población y presencia/ausencia, en función de los tratamientos de control y el Testigo, puesto que una vez analizados los datos por técnica estadística univariada se encontraron valores de media sin separación con nivel de confianza del 95\%, por cuanto se acepta la hipótesis nula del estudio.

Existe diferencia significativa en la severidad del daño causado por P. longifila cuando se analiza la variable frutos sanos/afectados, en función de los Tratamientos 2 y 3, que contienen ingredientes de síntesis química, y el Tratamiento 1 y Testigo, los cuales contienen otro tipo de ingredientes o no contienen ninguno; puesto que una vez analizados los datos por técnica estadística univariada se encontraron valores de media separados con un valor de $\mathrm{p}<0,05$, y por tanto se rechaza la hipótesis nula.

Los tratamientos más efectivos son el tratamiento 3 (químico) y el tratamiento 2 (químicorepelente) los cuales presenta menor severidad de daño, así mismo se obtiene una mayor cantidad de frutos sanos y longevidad de la planta durante la etapa productiva. 


\section{LITERATURA CITADA}

1. Álvarez-Hernández, J. C. (2012). Comportamiento agronómico e incidencia de enfermedades en plantas de tomate (Solanum lycopersicum L.) injertadas. Palmira: Acta Agron. vol.(61) $\begin{array}{llll}\text { no.2. } & \text { pp. } & \text { Recuperado }\end{array}$ http://www.scielo.org.co/scielo.php?script=sci_arttext\&pid=S0120-28122012000200004

2. ANDI. (2015). manual para la elaboración de protocolos para ensayos de pqua. Bogota D.C. Recuperado de http://www.andi.com.co/es/PC/SobProANDI/Documents/Ensayo/Manual\%20protocolos\%20 ensayos\%20eficacia\%20PQUA.pdf

3. Ayqui, S. y Sánchez, G. (1994) Biología y comportamiento de Prodiplosis logifila Gagné (Diptera: Cecidomyiidae) en el cultivo de papa. Perú. P. 5 -16.

4. Bolaños Fernandez, H. (15 de Abril de 2016). Entrevista personal productor de tomate. (A. Bolaños Trujillo, Entrevistador)

5. Cardona Suarez, C. M., Cristóbal Yepes, F., \& Cotes Torres, J. M. (2010). EVALUACIÓN DE LA ROTACIÓN DE PLAGUICIDAS QUÍMICOS Y BIOLÓGICOS SOBRE Prodiplosis longifila Gagné Diptera: Decidomyiidae) EN TOMATE (Solanum lycopersicum L.). Revista Facultad de Ciencias Basicas, 6(1)

6. Chavés Vergara, J. A. (2002). Estudio de la dinamica poblacional de Prodiplosis longifila Gagné (Diptera: Cecidomyiidae) en el cultivo de tomate en al localidad de Lodana Manabi. Recuperado de https://books.google.com.co/books?id=vNoZAgAAQBAJ\&pg=PA1991\&lpg=PA1991\&dq= prodiplosis+longifila+entrada+a+Colombia\&source=bl\&ots=fHrZ3D_A8t\&sig=-DZpA- 
8e3bUAsvRX5TpbwaZ7my8\&hl=es\&sa=X\&ved=0ahUKEwjRtJCwybXMAhVIrB4KHXI8

AEQQ6AEIWDAN\#v=onepage\&q=prodiplosis\%20

7. DANE. (14 de 02 de 2014). Boletín Semanal PRECIOS MAYORISTAS. Obtenido de https://www.dane.gov.co/files/investigaciones/agropecuario/sipsa/Semana_8feb_14feb_2014. pdf

8. DANE. (2016). ENCUESTA NACIONAL AGROPECUARIA ENA 2015. Obtenido de https://www.dane.gov.co/files/investigaciones/agropecuario/enda/ena/2015/boletin_ena_201 5.pdf

9. Delgado, A. (1998). Biologìa y evaluación de métodos de manejo de Prodiplosis longifila Gagnè (Diptera: Cecidomyiidae) en el cultivo de tomate Lycopersicon esculentum del valle del cauca. (Tesis de ing. Agrónomo). Fac. de ciencias agropecuarias. Universidad Nacional de Colombia. palmira, Colombia

10. Diaz-Silva, F. F. Aspectos agroecológicos para el Manejo Integrado de Prodiplosis longifila Gagné en la irrigación Chavimochic. Trujillo, Peru: Escuela de Ciências Biológica Universiudad Nacional de Trujillo (UNT), 2011. 94p.

11. Di Rienzo J.A., Casanoves F., Balzarini M.G., Gonzalez L., Tablada M., Robledo C.W. InfoStat versión 2017. InfoStat Group, Facultad de Ciencias Agropecuarias, Universidad Nacional de Córdoba, Argentina. URL http://www.infostat.com.ar

12. Fernández-Kohatsu., E. (2016). Comparativo de insecticidas para el control de Prodiplosis longifila Gagné (Diptera : Cecidomyiidae) en el cultivo de papa (Solanum tuberosum) Var. $\begin{array}{llll}\text { Canchán. } & \text { Lima-Perú. } & \text { Recuperado de }\end{array}$ http://repositorio.lamolina.edu.pe/bitstream/handle/UNALM/2632/H10-F4737T.pdf?sequence $=1$ 
13. Hernandez, L. M., Guzman, Y. C., Martínez-Arias, A., Manzano, M. R., \& Selvaraj, J. J. (2015). The bud midge Prodiplosis longifila: Damage characteristics, potential distribution and presence on a new crop host in Colombia. SpringerPlus, 4(1), 205

14. Lozano, J. (09 de Octubre de 2016) Entrevista personal Ingeniero Agrónomo. (M. Muriel Rodríguez, Entrevistador)

15. Mena Pérez, Y. M. (2012). Evaluación de la resistencia a Prodiplosis longifila Gagné (diptera: cecidomyiidae) en materiales segregantes de tomate cultivado y accesiones silvestres de Solanum habrochaites Knapp y Spooneer var. glabratum. (tesis de posgrado). Recuperado de http://www.bdigital.unal.edu.co/9165/1/yurimercedesmenaperez.2012_-_Ok.pdf

16. Mena Pérez, Y. M., Mesa, N. C., Estrada, E. I., \& García Valencia, Y. (2012). Evaluación de la resistencia a Prodiplosis longifila Gagné (Diptera: Cecidomyiidae) en genotipos de tomate cultivados y silvestres. Acta Agronómica, vol. (63), núm. 2, 2014, pp. 1-13. Recuperado de http://www.bdigital.unal.edu.co/31107/1/30210-201851-1-PB.pdf

17. OIT. (2000). Seguridad y salud en la agricultura. GINEBRA: SafeWork,. Recuperado de http://www.ilo.org/wcmsp5/groups/public/---ed_protect/---protrav/--safework/documents/publication/wcms_117460.pdf

18. Posada, R. H.. (2016). Patrones de distribución de insectos de escama sobre 4 especies del arbolao urbano de Bogotá, Colombia. Revista de Investigación Agraria y Ambiental. Volumen 7, No. 1. Universidad Nacional Abierta y a Distancia - UNAD. Bogotá, Colombia.

19. Rendón-Torres, L. Y. (2015). Control químico de Prodiplosis longifila en el cultivo de tomate (Solanum lycopercicum mill.). Guayaquil-Ecuador: Universidad de Guayaquil. Recuperado de http://repositorio.ug.edu.ec/handle/redug/7390Rivera, A. (12 de Mayo de 2016). Entrevista personal Productor de Tomate. (A. Bolaños Trujillo, Entrevistador) 
20. Rincón, C. (09 de Octubre de 2016) Entrevista personal Asistente Técnico. (M. Muriel Rodríguez, Entrevistador)

21. Sanmiguel, D. (2016). PLAN DE DESARROLLO GIGANTE AMABLE CONSTRUYENDO PAZ, REQUISITO DE LEY O PROYECTO DE DESARROLLO PARA GIGANTE HUILA Bogotà: Universidad Militar Nueva Granada. Recuperado de http://repository.unimilitar.edu.co/bitstream/10654/14849/1/Diego\%20Mauricio\%20Sanmigu el\%20PascuasArticulo2016.pdf

22. Sarmiento, Jorge. (1997). Manejo de Prodiplosis longifila Gagné. Perú. Pág.1-4.

23. Valarezo , O., Canarte, E., Navarrete, B., \& Arias, M. (2003). Prodiplosis Longifila. Recuperado https://books.google.com.co/books?id=94EzAQAAMAAJ\&pg=PA17\&lpg=PA17\&dq=morf ologia+de+prodiplosis+longifila\&source=bl\&ots=rDo8Hd9aos\&sig=_tMhpPOyV9QNOB47 mc5a9sICwp8\&hl=es\&sa=X\&ved=0ahUKEwifjLTz3ofOAhXKXR4KHZiXBTw4ChDoAQ gZMAA\#v=onepage \&q\&f=false

24. Vélez Salazar, B. P. (1998). Biología, comportamientos y hospederos de Prodiplosis longifila (Diptera: cecidomyiidae) en el cultivo de tomate. (tesis de pregrado). Recuperado de https://books.google.com.co/books?id=V38zAQAAMAAJ\&pg=PR2\&lpg=PR2\&dq=prodipl osis+longifila\&source $=$ bl\&ots $=$ YcJwKwVlu\&sig=yDBkZeq9EYKVCLxfh9VyysvgyF8\&hl=es\&sa=X\&ved=0ahUKEwjvgP_Ywrb MAhXIbR4KHbiYBYk4ChDoAQhXMAk\#v=onepage\&q\&f=false 